\title{
Observation of the Effectiveness of Comprehensive Therapy for Cervical Disposition with Health Exercise for Cervical Spine in Coordination with Medical Massage
}

\author{
Liangbing Yang, Haikuan Wang, Ying Shao \\ Guangzhou University of Chinese Medicine, Guangzhou Guangdong, 510006, China
}

Keywords: Health exercise for cervical spine, Medical massage, Cervical disposition, Therapeutic effect.

\begin{abstract}
Purpose: to analysis the effectiveness of health exercise for cervical spine in coordination with medical massage. Method: observing approach, 90 cases with cervical disposition are chosen as research subjects, and randomly divided into two group of 45 to 45; comparison are made between the control group applied only with medical massage while the observation group applied both medical massage and health exercise for cervical spine. Result: before the therapy, the cervical disability index for both groups are $(42.15 \pm 8.22)$ for control group and (41.96 \pm 8.19$)$ for observation group, with no clear differences; while visual analogue scores for both groups are(7.59 \pm 3.02$)$ (7.75 \pm 1.85 ) with no clear differences, the $\mathrm{P}>0.05$; after the therapy, for the control group the scores are the cervical disability index (21.03 \pm 2.47$)$, visual analogue score $(4.19 \pm 1.64)$; the observation group the scores are(32.58 \pm 2.17$)$ and $(5.11 \pm 1.94)$; both groups had achieved pretty good curative effective, the figures in observation group are sensibly lower than the control group $\mathrm{P}<0.05$. the result for overall response rates are observation group 97.78\%, control group 82.22\%, observation group obviously higher than the control group $\mathrm{P}<0.05$. Conclusion: the comprehensive therapy with the additional health exercise for cervical spine and basic medical massage can conduct further relieve for symptoms of cervical disposition, have multiple significant effect on functions and effectiveness of cervical vertebra disease, have high clinical value and can be extended.
\end{abstract}

\section{Preface}

Cervical vertebra disease is one of the most common diseases currently, which is a degenerative disease in cervical disc and sounding ligaments; generally speaking, cervical vertebra disease can cause sustainable symptoms like numbness and pain in lesion local nerve, muscles, blood vessels and soft tissues of all kinds, even dizzy in serious condition which can made extremely influence to the quality of life. ${ }^{[1-5]}$ According to its varieties, cervical vertebra disease can be divided into cervical disposition, cervical radiotelegraphy, Vertebral artery type of cervical disposition, mixed type of cervical disposition ,etc. non-surgical treatments are main clinical treatment for cervical vertebra disease , including drug therapy, cervical spine immobilization therapy, acupuncture therapy and medical massage ,etc. this research achieved a satisfying conclusion by practical comparison of the different therapies, between the results of medical massage and comprehensive therapy with health exercise for cervical spine,. The research is shown as below.

\section{Materials and Methods}

\section{General information.}

90 cases of patients with cervical disposition from our hospital January 2014 to January 2016 are chosen as subjects; they all marked with numbers and randomly divided into 45 cases in observation group and 45cases in control group; both the patients and family numbers have adequate knowledge of purpose and content of the research, and signed the informed content form; the ethic commission of our hospital endorsed this research. The diagnoses and observation of 90cases all match the diagnostic criteria in Diagnosis and treatment and rehabilitation Guide for cervical vertebra disease, the patients are suffering from varying degrees of pain in back, neck and shoulders, and identified 
tender point by clinical examinations. The general information for the two groups are:,observation group have20cases of male patients and 25cases of female; age groups are between 69 to 28, the average age is (49.21 \pm 6.36$)$. the control group has 22cases male patients and 23 female; the age group are between 70 to $29 \mathrm{~m}$ with average age $(48.13 \pm 6.06)$. the exclusion criteria for this research are: (1) high blood pressure patients; (2) coronary heart disease patients; (3) congenital malformations patients; (4)bone tumors patients; (5) patients with serious visceral disease; (6)frozen shoulder patients; (7) patients With Rheumatic muscle fiber; (8) patients suffer from neurasthenia; (9) patients severe bone hyperglycemia symptom; (10) Scoliosis deformity patients. From the causes of the cervical disposition, observation group patients have 18 cases caused by bad habits, of which 17cases are by bad sleeping postures, and 10cased caused by trauma; control group patients have 20cased caused by bad habits, of which 17 cases by bad sleeping postures and 8cases of trauma. There is no obvious differences between observation group and control group patients in general information, $\mathrm{P}>0.05$ can be compared.

\section{Therapeutic methods.}

Control group: medical massage are taken for the 45 cases in this group. First of all, the patient is in sitting position, medical massages based on rolling technique is done in his/her shoulder neck and back to relaxing, this treatment last for $10 \mathrm{~min}$. secondly, based on the tender part of the patient, locating points technique in radiation directions was taken, press and rub continuance in FengFu , Yamen and Dazui acupuncture points, then press and rub in Fengchi and Dashu points besides the last three points are Tianzhu , Jianzhong, and Shousanli., keep pressing until acid bilge feeling appeared, finally, holding the occidental part and the lower mandible of the patient with both hands, pulling upwards slowly by suitably strength, then twisting at the same speed; the chapter need to repeat 20 times the period last for 7days.

Observation group: In addition to the medical massage of the control group, the 45 cases in this group, the health exercise for cervical spine added for co-operation. Concrete steps are: (1)patient is in standing position, meanwhile posture both hands on hips with shoulder and feet in width apart and relaxing, keep head up, take deep breathing with as the guided. (2) Once the breathing practice is done, do antioxidant and retro extension action, begin with repeating "looking up - back-looking down-back"10-15 times according to the patients. (3) do the left and right retrogression, just breathing at the same time, , breath in to the left breath out to return, breath in to the right and out to return; now switch to left and right, fitting the breathing, repeat 10-15 times according to the patients. (4) then do the chest up and rocker exercises. First bucking the delete attributes of both hands, and trying to reach shoulder o the other side with finger, with outwards exposition of delete attributes; finally shaking shoulder backwards, with very slow speed, to avoid injury. repeat 10-15 times according to the patients.(5) next chapter is for the rounded resistance, patient is in standing position with head looking forward and up, try to keep the head steady in the position and resist the pushing strength from both hands on forehead and e head backwards by both hand in forehead, until acid bilge feeling shown at the neck muscles; then put one hand in the forehead pushing to "left to right: "right to left" motion, while the muscles laid resistance, all l the four direction is one chapter, repeat 10-15 time according to the patient.(6) the final chapter is upward motion from occidental region. First of all, craning neck forwards by $15^{\circ}$, looking down while bending both elbows and surrounding occidental region; then pull the head upwards by both hand-back, repeat this chapter 8-10times according to the patient, keep still between exercises by 3-5 second. Al the above chapter to have to carried on a slow ,suitable, progressive, orderly and evenly principles, prevent from injures; moreover, minimized the motion until sense of tension appear do not overdue nor snap motion $\mathrm{m}$, and all the actions have to match with breathing. This health exercise for cervical spines should be only carried under the supervision of doctors, once a day 7days a period, and monitoring the effect and relative figures. 


\section{Observation measures index.}

The research uses three indexes for measure the results of treatment of the patient including effectiveness, visual analogue score and the cervical disability index. Of which, the target for visual analogue score (VAS)is the score for the pain level tension location, start from 0 to 100 , the score is proportional to the pain level, higher the score more painful may be felt; cervical disability index (NDI) targeted the ability of daily life and the clinical symptoms, including 10 items, each item score form 0-5, the score is proportional to cervical disability level, higher the score more difficulty the patient will have. More over the effectiveness of the treatment can be scaled as cured, markedly effective, effective and in-effective four phrases, cure means the patient can carry daily normal activities, clinical symptom disappeared, markedly effective means the clinical symptom has improved dramatically, discomfort intermittently appear, but have no impact to daily life, effective means symptoms have been relived in certain level, lifting capacity have improved while sharp pain still remains, clinical symptom made no improvement even worse ${ }^{[6-9]}$.

\section{Statistical processing.}

All the data for this research is operated by software SPSS19.0. of witch, T score is use for measure mental data, $\mathrm{x}^{2}$ score is used in numeration data, $\mathrm{P}<0.05$ means the differences are was statistically significant.

\section{Result}

\section{Compartment of the relative clinic index.}

As the result shown, there is no obvious differences on the scores before the treatment between observation group and control group, andP $>0.05$ in cervical disability index and visual analogue score; the scores after treatment have achievement great improvement compare to before and $\mathrm{P}<0.05$; the scores of observation group are significantly lower than the control group, and $\mathrm{P}<0.05$. details as shown in table 1.

Table 1. Compartment of relative clinical index between observation group and control group patients ( $\overline{\mathrm{X}}_{ \pm \mathrm{s}}$ )

\begin{tabular}{ccccc}
\hline Group & \multicolumn{4}{c}{ Cases Treatment stage cervical disability index visual analogue score } \\
\hline \multirow{2}{*}{ observation group } & \multirow{2}{*}{45} & Before & $(42.15 \pm 8.22)^{*}$ & $(7.59 \pm 3.02)^{*}$ \\
\cline { 3 - 5 } & & After & $(21.03 \pm 2.47)^{* *}$ & $(4.19 \pm 1.64)^{* *}$ \\
\hline \multirow{2}{*}{$\mathrm{t}$} & & 5.07 & 2.21 \\
\hline \multirow{2}{*}{$\mathrm{p}$} & \multirow{2}{*}{45} & Before & $(41.96 \pm 8.19)$ & $(7.75 \pm 1.85)$ \\
\cline { 3 - 5 } control group & After & $(32.58 \pm 2.17)$ & $(5.11 \pm 1.94)$ \\
\hline \multirow{2}{*}{$\mathrm{t}$} & & & 2.26 & 2.73 \\
\hline $\mathrm{p}$ & & & $<0.05$ & $<0.05$ \\
\hline
\end{tabular}

Note :*No big difference with control group and $\mathrm{P}>0.05$; **significantly lower than the control group and $\mathrm{P}<0.05$.

\section{Comparament of effectiveness before and after treatment.}

As shown in the research, the overall effectiveness of the treatment for observation group patient is $97.78 \%$,control group is $82.22 \%$, the observation group significantly higher than control group ,and $\mathrm{P}<0.05$. detail as shown on table 2 .

Table 2. The compartment effectiveness between observation group and control group patient (n/\%)

\begin{tabular}{ccccccc}
\hline Group & $\mathrm{n}$ & ineffective & effective & , markedly effective & cured & overall effectiveness \\
\hline observation group & 45 & $1(2.22)$ & $5(11.11)$ & $15(33.33)$ & $24(53.33)$ & $44(97.78)$ \\
\hline control group & 45 & $8(17.78)$ & $20(44.44)$ & $7(15.56)$ & $10(22.22)$ & $37(82.22)$ \\
\hline x2 & 6.05 & 12.46 & 3.85 & 9.26 & 6.05 \\
\hline $\mathrm{P}$ & & $<0.05$ & $<0.05$ & $<0.05$ & $<0.05$ & $<0.05$ \\
\hline
\end{tabular}




\section{Discussion}

Cervical disposition is one of the clinical common type of cervical vertebra disease, it is the early stage of the cervical vertebra disease, is the clinic majority with cervical vertebra disease. non-surgical treatments are main clinical treatment for cervical vertebra disease, including acupuncture therapy and medical massage ,etc . medical massage is the preferred therapy for cervical disposition ,it can restore basic cervical function by relaxing neck and shoulder muscles, and help patient dispel wind, relieve pain and activate muscle by pressing and rubbing relative acupuncture points; at present health exercise for cervical spine as a new rehabilitation method is available, and proved that it do have some effect for the cervical disposition. ${ }^{[10-15]}$ in order to future study its practice value, this article conducted a compartment research between medical massage comprehensive therapy with health exercise for cervical spine for cervical disposition. The research achieved satisfying conclusion.

The patients is the main subject for health exercise for cervical spine, through the orderly guiding, his/her neck will complete a serious suitable activities by pulling muscles in back, shoulder and neck to press around acupuncture points in shoulder and neck region, and to achieve the target of restoring some basic function. According to this research, the scores of observation group are significantly lower than control group in cervical disability index and visual analogue score and $\mathrm{P}<0.05$; it concluded that with the assistants of health exercise for cervical spine, patients cervical vertebra disease clinical symptom living standard of the patient of the patient can be future improved. Beside the effectiveness of observation group is also obviously higher than control group, which also approve that the practical value of the health exercise for cervical spine.

In summary, the comprehensive therapy for cervical disposition patients with health exercise and medical massage can contribute to relive clinic symptom and restore the function and life quality, it is a therapy with remarkable curative effect, can be popularized .

\section{Acknowledgments}

Fund Project: innovation school of engineering, Guangzhou University City elected course the Medical Health Care Massage teaching mode optimization research, NO.:A1-AFD015151Z1514.

\section{References}

[1] Zhao Fanping, Wang Chongmiao, Gu Wenye et al. Massage medical exercise health cervical for spine disease, Cervical vertebra International Journal of traditional Chinese medicine, 2014,36 (09):802-806.

[2] Zhao Fanping, Wang Chongmiao, Zhang Yifei et al. Massage medical combined with health care operation in the treatment of cervical type, nerve root type vertebra disease, Cervical Jilin Chinese medicine, 2014,34 (09):951-955.

[3] Wei Hanxian, Jin Guo Zheng, Huang Bristol. Comprehensive therapy of traditional Chinese medicine in the treatment of vertebral artery type cervical vertebra disease curative effect observation, Massage and rehabilitation medicine, 2014,5 (12):78-80.

[4] Liang Yijun, Zhang Lincan exercise.Health for cervical spine with massage medical treatment disposition cervical effect observation, China Rural Medicine, 2016,23 (02):41-42.

[5] Tang Sen, Luo Xiangyun, Shi Qin Da et al. The therapeutic effect of electroacupuncture at the point of the acupuncture points in the treatment of disposition, Cervical Shanghai Journal of acupuncture and moxibustion, 2014,33 (09): 840-842

[6] Zhong Minying, He Qingtao, Wu Siping et al. The effect of floating acupuncture combined with Stretch Therapy on the treatment of disposition, Cervical Journal of practical medicine, 2015,31 (08):1340-1342. 
[7] Shen Tong, Li Li, Wu Zeyong, et al. Clinical research on the treatment of disposition cervical with the combination of exercise therapy and manipulation, Chinese Journal of rehabilitation medicine, 2014,29 (09):860-862.

[8] Zhao Minghua. The fire needle therapy effect on cervical spondylosispatients neckpain, light 2014,29 Chinese medicine, (07):1451-1453.

[9] high rock, Zhao Runchen. Zhao elastic dial collateral method in the treatment of cervical disposition experience analysis, World of traditional Chinese medicine and Western medicine combined with the magazine. 2015,10 (12):1669-1671.

[10]Cui Shaoyang, Xu Mingzhu, Lai Xin Sheng et al. Abdominal acupuncture combined with intermediate frequency pulse electrical therapy spondylosispatients, clinical observation cervical Chinese traditional Chinese medicine emergency, 2015,24 (04):702-704.

[11]Liu Hongju, Qiu Yunfeng, Dong Ling et al. The effect of suspended training combined with intermediate frequency electric treatment on disposition, Cervical Journal of Guiyang Medical University, 2015,40 (06):638-643.

[12]Liao Xiao, Ma Huiyi, Mu Jing et al. Back to the medical reinforcement therapy in the treatment of cervical disposition clinical curative effect, Journal of Ningxia Medical College, 2015,37 (08):861-864

[13]Marden Shang, Pang tsan. Trapezius acupuncture suspended point for the treatment of cervical disposition clinical efficacy evaluation, Journal of clinical acupuncture and moxibustion, 2015,31 (02):18-20.

[14]Li Yong. Abdominal acupuncture and cupping therapy for disposition, clinical observation cervical Chinese traditional medicine technology, 2014,21 (z2):11.

[15]Li Donghong, Gao Shuang, Zhang Wei et al. The theory of "moving the sun" to guide the treatment of juvenile disposition, Cervical Journal of Changchun University of Traditional Chinese Medicine, 2015,31 (02):313-315. 